EPJ Web of Conferences 21, 06001 (2012)

DOI: $10.1051 /$ epjconf/20122106001

C Owned by the authors, published by EDP Sciences, 2012

\title{
Fusion probability and survivability in estimates of heaviest nuclei production
}

\author{
Roman N. Sagaidak ${ }^{*}$ \\ Flerov Laboratory of Nuclear Reactions, Joint Institute for Nuclear Research, 141980 Dubna, RF
}

\begin{abstract}
Production of the heavy and heaviest nuclei (from Po to the region of superheavy elements close to $Z=114$ and $N=184$ ) in fusion-evaporation reactions induced by heavy ions has been considered in a systematic way within the framework of the barrier-passing model coupled with the statistical model (SM) of de-excitation of a compound nucleus $(\mathrm{CN})$. Excitation functions for fission and evaporation residues (ER) measured in very asymmetric combinations can be described rather well. One can scale and fix macroscopic (liquid-drop) fission barriers for nuclei involved in the calculation of survivability with SM. In less asymmetric combinations, effects of fusion suppression caused by quasi-fission (QF) are starting to appear in the entrance channel of reactions. QF effects could be semi-empirically taken into account using fusion probabilities deduced as the ratio of measured ER cross sections to the ones obtained in the assumption of absence of the fusion suppression in corresponding reactions. SM parameters (fission barriers) obtained at the analysis of a very asymmetric combination leading to the production of (nearly) the same $\mathrm{CN}$ should be used for this evaluation.
\end{abstract}

\section{Introduction}

Complete fusion between an accelerated heavy ion and heavy target nucleus is defined as their amalgamation into a compound system inside the fission barrier. This process is unambiguously identified by the direct observation of evaporation residues (ER) produced after cooling of an excited compound nucleus $(\mathrm{CN})$ by light particles (neutrons) evaporation. Complete fusion reactions have been successfully used in synthesis of the heaviest elements at the border of nuclear stability in experiments performed during the last 20 years [1,2]. Such experiments are extremely challenging as the formation of heavy/superheavy ER is heavily suppressed not only by the CN-fission, but also by a non-equilibrium process called quasi-fission (QF) [3-7]. Following the capture of a projectile and target nuclei, a system may re-separate prematurely, not forming a true CN. Such events represent the transition between deep-inelastic collisions (DIC) and complete fusion. In DIC the entrance channel mass-asymmetry is preserved, but there can be large dissipation of kinetic energy and angular momentum [8]. CN formation, in contrast, is characterized by equilibration of all degrees of freedom, and hence completes loss of identity of the entrance channel. Intermediate

*e-mail : sagaidak@nrmail.jinr.ru

This is an Open Access article distributed under the terms of the Creative Commons Attribution-Noncommercial License 3.0, which permits unrestricted use, distribution, and reproduction in any noncommercial medium, provided the original work is properly cited. 
between DIC and $\mathrm{CN}$ fission, QF has full energy dissipation but incomplete drift toward the energetically favoured mass-symmetric configuration $[5,7]$.

Intensive studies of the mechanisms of complete fusion in fission experiments [4, 6, 7] and in experiments on ER production [9-12] in reactions leading to heavy and very heavy composite systems, make it evident that the growing QF effect leads to a rapid decrease in the fusion probability and consequently to the drop of production cross sections for the heaviest elements as their atomic number is increased. Such dependence is clearly seen in the case of 'cold' fusion reactions [1]. One of a qualitative explanation of the fusion suppression effect was proposed in the framework of the liquid drop (LD) model [11]. It is suggested that the transition from the contact configuration to the $\mathrm{CN}$ configuration is determined by a presence of the conditional barrier along the mass-asymmetry coordinate [13] and the entrance-point position with respect to the top of the barrier, i.e., relatively to the Businaro-Gallone (BG) point [14]. The Generalized Liquid-Drop Model (GLDM) [15] calculations of the potential energy surface (PES) in the $(Z, N)$-plane for the contact configuration of spherical nuclei give us an examination of the PES for the ${ }^{216} \mathrm{Ra}^{*} \mathrm{CN}$ in more detail (see Figure 1) than considered in [11]. The calculations take into account proximity energy [16]. The entrance points for asymmetric combinations (with Ar and lighter projectiles) lay well above the bottom of the valley (minima of the potential energy corresponding to the driving potential), on mountainsides of the PES. Shell corrections [17] strongly modulate the PES and a driving potential. In the framework of this approach fusion with ${ }^{48} \mathrm{Ca}$ and ${ }^{86} \mathrm{Kr}$ could be considered as the suppressed one, since their entrance points lay on the bottom of the valley, in front of the conditional barrier on a way along the mass-asymmetry coordinate to complete fusion as shown in Figure 1. In the ${ }^{48} \mathrm{Ca}+{ }^{168} \mathrm{Er} \rightarrow{ }^{216} \mathrm{Ra}{ }^{*}$ experiment fusion suppression is observed and it corresponds to the fusion probability $P_{f u s} \approx 0.3$ [12]. The value deduced by comparing measured ER cross sections with those obtained in the ${ }^{12} \mathrm{C}+{ }^{204} \mathrm{~Pb} \rightarrow{ }^{216} \mathrm{Ra}^{*}$ reaction $[11,12]$.

A number of models using PES in calculations were developed to describe complete fusion of massive nuclei leading to production of heavy and heaviest nuclei. Among them there are calculations based on a di-nuclear system (DNS) concept (see, e.g., results of calculations in [18, 19]). Other ones use a two-centre model approach [20]. One should mention that these models use

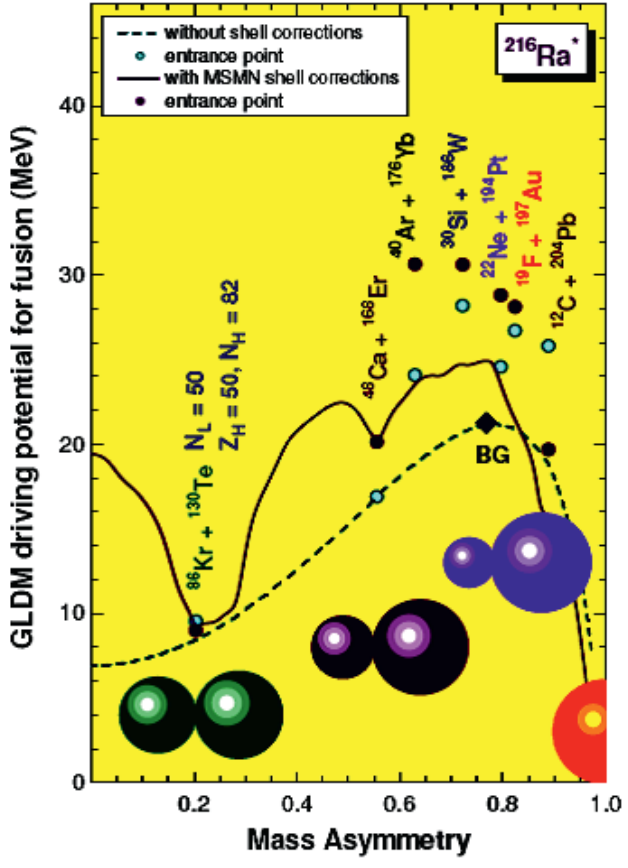

Fig. 1. Driving potential extracted from the PES obtained in the framework of GLDM $[15,16]$ for the ${ }^{216} \mathrm{Ra} \mathrm{CN}$ as a function of the mass asymmetry (solid and dashed lines corresponding to the calculations with and without shell corrections [17], respectively). Filled and open circles correspond to the positions of the entrance points on PES for some combinations, which are also calculated with and without shell corrections [17], respectively.

more sophisticated multi-dimensional PES with taking into account a distance between reactants, their static and dynamic deformations, a relative angular momentum, etc (see, e.g., [21]). In calculations, the resulting ER cross section can be parameterized as follows:

$$
\sigma_{E R}=\sum_{L=0}^{L_{\max }} \sigma_{\text {cap }}(E, L) P_{\text {fus }}(E, L) W_{\text {sur }}\left(E_{C N}^{*}, L\right),
$$

where $\sigma_{\text {cap }}$ is a capture cross section as a function of the c.m. energy $E$ and angular momentum $L$, and $W_{\text {sur }}$ is survivability of nuclei in the evaporation-fission process of the $\mathrm{CN}$ decay at the corresponding excitation energy $E_{C N}^{*}$ and angular momentum $L$. Despite of variety of theoretical models they 


\section{$\mathrm{CNR} * 11$}

demonstrate rather good agreement of calculated ER cross sections with experimental ones. This agreement is shown, e.g., in the analysis [22] using a number of models that result in the calculated cross sections for the heaviest ER produced in 'cold' fusion reactions [1]. At the same time, this analysis shows a large spread in the $P_{\text {fus }}$ values given by these models in calculations (a difference exceeds two orders of the value). To determine $P_{f u s}$ strictly the authors [22] measured the capture cross section and extracted the $\mathrm{CN}$-fission component with the decomposition of measured angular distributions for fission-like fragments produced in the ${ }^{50} \mathrm{Ti}+{ }^{208} \mathrm{~Pb} \rightarrow{ }^{258} \mathrm{Rf}$ reaction. Such procedure is not completely model-independent one since it implies some constraints on the angular momenta and moments of inertia inherent in the $\mathrm{CN}$-fission and QF processes [6].

In a contrast to the previous study with the decomposition of angular distributions integrated over a mass and energy of fission-like fragments [22], in our study of the ${ }^{48} \mathrm{Ca}+{ }^{154} \mathrm{Sm} \rightarrow{ }^{202} \mathrm{~Pb}^{*}$ reaction the $\mathrm{CN}$-fission component was extracted from measured mass-angular distributions with taking into account a total kinetic energy (TKE) of fission fragments (FF) [23]. With these constraints, FF of the masses in the region of $A_{C N} / 2 \pm 20$ only had a symmetric angular distribution in the c.m. system. These FF may correspond to 'true' CN-fission. Therefore, the fraction of the QF component and the corresponding $P_{\text {fus }}$ values could be 'definitely' extracted [23]. Nevertheless a comparison of the ER cross sections measured in this reaction [24] with those obtained in ${ }^{16} \mathrm{O}+{ }^{186} \mathrm{~W}$ leading to the same ${ }^{202} \mathrm{~Pb}^{*} \mathrm{CN}$ shows distinctly lower values of the fusion probability. This comparison (together with the calculated $P_{\text {fus }}$ values obtained in the framework of the DNS concept [25]) is shown in Figure 2. The question arises: why is there the difference in the $P_{\text {fus }}$ estimates obtained with the comparison of the ER data and from fission experiments? One should mention that in our experiments FF and ER were detected in the same runs following the same normalization procedure based on Rutherford scattering for the cross section estimates. At the same time, within our data processing procedure, we could i) underestimate quantity of $\mathrm{QF}$ events (using simple cutting and Gaussian fits) because of the interference of QF events with deep-

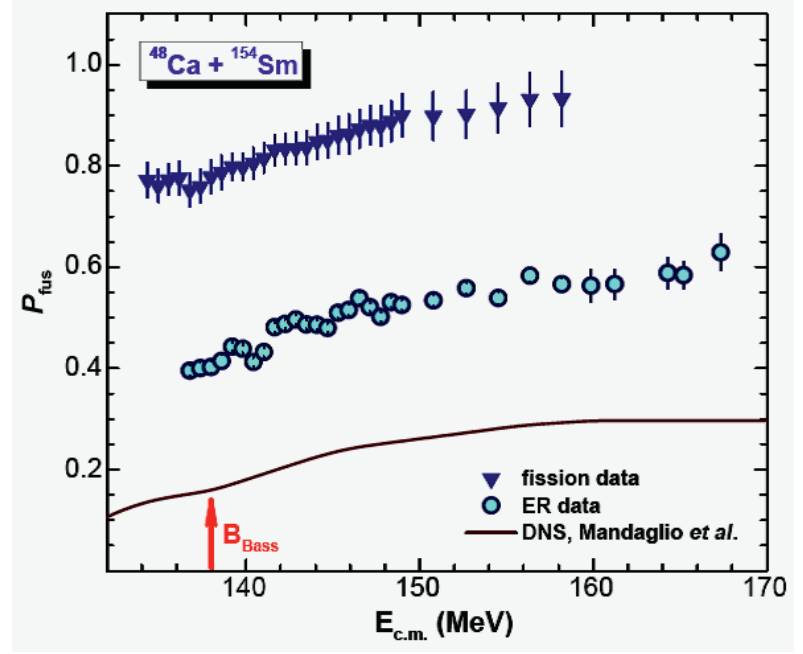

Fig. 2. Fusion probabilities extracted from the fission data obtained in the ${ }^{48} \mathrm{Ca}+{ }^{154} \mathrm{Sm} \rightarrow{ }^{202} \mathrm{~Pb}^{*}$ reaction [23] and similar values derived from the comparison of the ER cross sections measured in the same reaction with those obtained in ${ }^{16} \mathrm{O}+{ }^{186} \mathrm{~W} \rightarrow{ }^{202} \mathrm{~Pb}^{*}$ [24] (symbols). $P_{\text {fus }}$ calculated in the framework of the DNS concept [25] is also shown by solid line for comparison.

inelastic ones and ii) overestimate quantity of $\mathrm{CN}$-fission events corresponding to a 'right' variance of the mass distributions and to the symmetric mass-angular distributions. The latter implies some equilibration and compactness of a system near the saddle point without passing over the fission barrier, i.e., without 'true' $\mathrm{CN}$ formation. So fission data may not give us adequate values of the fusion probability whereas the detection of ER is an unambiguous signature of $\mathrm{CN}$ formation, as mentioned above.

\section{Method of analysis}

One can propose two empirical approaches for the extraction of the fusion probability values from ER cross section data. In the first one, ER cross sections measured in the reaction under study are compared to those obtained in a very asymmetric combinations leading to the same $\mathrm{CN}$. For the 
latter a lack of fusion suppression at energies above the Coulomb barrier is assumed, i.e., $P_{f u s}=1$. Bearing in mind that in the framework of a barrier passing (BP) model the ER cross section can be written as

$$
\sigma_{E R}=\pi / k^{2} \sum_{L=0}^{L_{\max }}(2 L+1) T_{L}(E, L) P_{f u s}(E, L) W_{s u r}\left(E_{C N}^{*}, L\right),
$$

where $k$ is a wave number and $T_{L}$ is a transmission probability of the potential-barrier passing for $L$ partial wave, which is about 1 at energies well above the barrier and survivability is the same for the same $\mathrm{CN}$; one can came to a simple relation for the estimates of the fusion probability

$$
P_{f u s}=\sigma_{E R}^{\text {expt }}\left(E_{C N}^{*}\right) k^{2} / \sigma_{E R}^{\text {asym }}\left(E_{C N}^{*}\right) k_{\text {asym }}^{2},
$$

where the terms with super-scripts asym correspond to the values measured and/or calculated for a very asymmetric combination for which $P_{f u s}=1$ is assumed.

In the case, when the capture cross section for the reaction under study is known, the formulae (1) and (2) allow to estimate the fusion probability as follows

$$
P_{f u s}=\sigma_{E R}^{\text {expt }}\left(E_{C N}^{*}\right) / \sigma_{E R}^{\text {calc }}\left(E_{C N}^{*}\right)^{\prime}
$$

where the calculated ER cross section $\sigma_{E R}^{c a l c}$ is based on the fitted capture cross section which is assumed to be equalled to the fusion cross section $\left(P_{f u s}=1\right)$ and can be calculated as

$$
\sigma_{\text {cap }}=\sigma_{f u s}=\pi / k^{2} \sum_{L=0}^{L_{\max }}(2 L+1) T_{L}(E, L) .
$$

A conventional barrier passing (BP) model that generally reproduces an experimental capture cross section as the barrier-passing one is used for the calculation of $\sigma_{E R}^{\text {calc }}$. The calculated BP cross section can be associated with the fusion cross section, implying for the fusion probability $P_{f u s}=1$. The effect of coupling the entrance channel to other reaction channels is taken into account phenomenologically via the fluctuations of the radius-parameter $r_{0}$. The radius-parameter fluctuations are generated with a Gaussian distribution around its average $r_{0}=1.12 \mathrm{fm}$ with the barrier fluctuation parameter $\sigma\left(r_{0}\right)$ [26]. These fluctuations simulate coupling effects [27] at energies around the Coulomb barrier to a degree sufficient for the purpose of the present analysis. All the BP model parameters are fixed with the exception of the strength $V_{0}$ and the relative barrier fluctuation parameter $\sigma\left(r_{0}\right) / r_{0}$ in the nuclear exponential potential [26]. Variations of these parameters allow one to reproduce the experimental cross-section data for fission and ER production at sub-barrier energies in calculations. Transmission probabilities for the potential barrier passing are calculated using the WKB approximation. Note that for strongly fissile compound nuclei the ER cross sections at energies well above the nominal fusion barrier [28] are weakly sensitive to the form of the nuclear potential and are mainly determined by the survivability calculated.

The survivability is calculated in the framework of statistical model approximations. The Reisdorf's expression [29] is used to calculate the macroscopic level-density parameters $\tilde{a}_{f}$ and $\tilde{a}_{v}$ in fission and evaporation channels, respectively. Macroscopic fission barriers adjusted with the scaling factor $k_{f}$ at the rotating LD fission barriers $B_{f}^{L D}(L)$ [30]. Therefore $k_{f}$ is the only fitting parameter in the expression determining a height of fission barrier:

$$
B_{f}(L)=k_{f} B_{f}^{L D}(L)-\Delta W_{g s} .
$$

The empirical masses [31] are used to calculate shell corrections $\Delta W_{g s}$ (as the difference between the empirical and LD masses [32]) as well as for the calculations of excitation and separation energies. The influence of the shell effects on the level density is included with an energy backshift in the lev- 


\section{$\mathrm{CNR} * 11$}

el-density parameter that is exponentially damped with increasing excitation energy. The same damping constant $E_{D}=18.5 \mathrm{MeV}$ was used as in a number of the previous works [9, 10, 12, 29].

The BP model and SM used in the present analysis are combined into the HIVAP code [26, 29] used for the calculations. Usually, in the analysis of the most asymmetric combinations, excitation functions at high energies can be fitted with the variation of $k_{f}$ mainly. The lack of any fusion suppression $\left(P_{\text {fus }}=1\right)$ is evident if ER and fission excitation functions are fitted with the same parameter values of the nuclear potential and $k_{f}$ in the whole range of excitation energies. In less asymmetric combinations the absence of the fusion suppression is not evident. Repeating aforesaid, the fusion probability can be obtained with the same scaling for fission barriers and other parameters of SM, as obtained in the analysis of the most asymmetric combination leading to the same $\mathrm{CN}[12,33]$.

\section{ER production in the vicinity of $\mathrm{N}=126$ shell}

In Figure 3 the reduced cross sections for ER produced in the ${ }^{16} \mathrm{O}+{ }^{186} \mathrm{~W}$ and ${ }^{48} \mathrm{Ca}+{ }^{154} \mathrm{Sm}$ reactions are compared $[24,33]$ with the aim to derive the fusion probability values. As we see, experimental values for the former exceed the ones for the latter by a factor of 1.5 at $E_{C N}^{*} \geq 70 \mathrm{MeV}$, where the effect of the fusion barriers [28] of both the reactions is negligible. The ER excitation function calculated for ${ }^{16} \mathrm{O}+{ }^{186} \mathrm{~W}$ reproduces the measured cross sections very well, whereas the similar one calculated for ${ }^{48} \mathrm{Ca}+{ }^{154} \mathrm{Sm}$ with the same parameters of SM as used in ${ }^{16} \mathrm{O}+{ }^{186} \mathrm{~W}$, but with the barrierpassing cross sections fitted to the experimental values corresponding to $\sigma_{c a p}=\sigma_{f i s}+\sigma_{E R}$, exceeds the measured ER cross sections by the same factor of 1.5 at $E_{C N}^{*} \geq 70 \mathrm{MeV}$. The excitation functions calculated for both the reactions are converged at $E_{C N}^{*} \geq 80$ $\mathrm{MeV}$ that implies formation of indeed the same $\mathrm{CN}$ at these energies. The ER excitation function calculated for ${ }^{48} \mathrm{Ca}+$ ${ }^{154} \mathrm{Sm}$ with the normalisation to $P_{\text {fus }}=0.57$ allows one to reproduce the experimental data at $E_{C N}^{*} \geq 60 \mathrm{MeV}$. The empirical $P_{\text {fus }}$ values shown in Figure 2 have been derived from the ratio of the measured ER cross sections to the calculated ones corresponding to the barrier-passing cross sections fitted to the experimental values of $\sigma_{c a p}=\sigma_{f i s}+\sigma_{E R}$ and to the survivability obtained in the ${ }^{16} \mathrm{O}+{ }^{186} \mathrm{~W}$ reaction. The latter is characterized by the scaling factor $k_{f}=0.85$ at the LD fission barriers for nuclei produced in the ${ }^{202} \mathrm{~Pb}^{*} \mathrm{CN}$ decay chains [33].

More severe fusion suppression is observed in reactions leading to more fissile $\mathrm{Th}^{*}$ compound nuclei. An example of data analysis is shown in Figure 4 for the reactions leading to the ${ }^{220} \mathrm{Th}^{*} \mathrm{CN}$. The data obtained in the very asymmetric ${ }^{16} \mathrm{O}+{ }^{204} \mathrm{~Pb}$ reaction can be reproduced with the corresponding scaling of the LD fission barriers. For the less asymmetric combination with ${ }^{40} \mathrm{Ar}$ and for the nearly symmetric one with ${ }^{124} \mathrm{Sn}$, ER cross

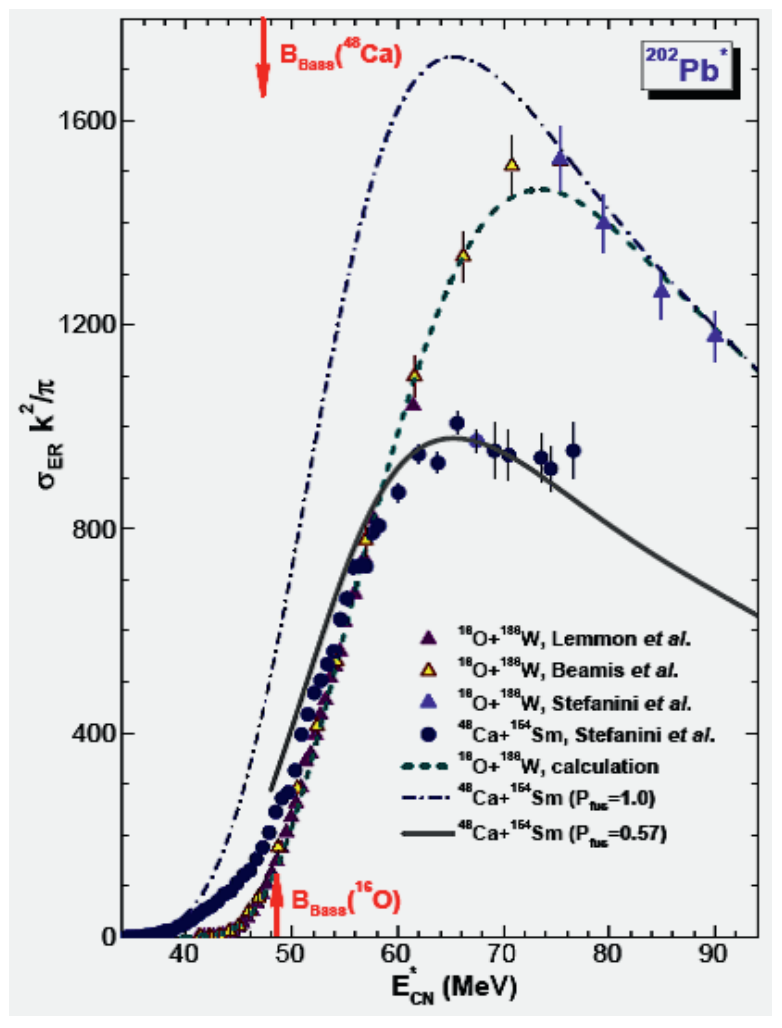

Fig. 3. Comparison of the reduced ER cross sections obtained for the ${ }^{16} \mathrm{O}+{ }^{186} \mathrm{~W}$ and ${ }^{48} \mathrm{Ca}+{ }^{154} \mathrm{Sm}$ reactions leading to the same ${ }^{202} \mathrm{~Pb}^{*} \mathrm{CN}$ [24, 33]. Experimental values and calculations are shown by different symbols and lines, correspondingly. See the text for further details. 
sections calculated with these barriers at energies well above the fusion barrier, exceed the experimental ones by a factor of 4 and 8, respectively. At sub-barrier energies, we obtain even stronger fusion suppression using barrier-passing cross sections corresponding to the capture cross sections fitted. For the nearly symmetric ${ }^{124} \mathrm{Sn}+{ }^{96} \mathrm{Zr}$ combination, one had to use the extra-extra-push (EEP) formalism with the parameters obtained in [7], giving the additional $\Delta B=17.1 \mathrm{MeV}$ to the potential barrier. This additive to the fusion barrier together with the normalisation of calculations with the $P_{f u s}=0.14$ value allow one to reproduce the ER cross sections.

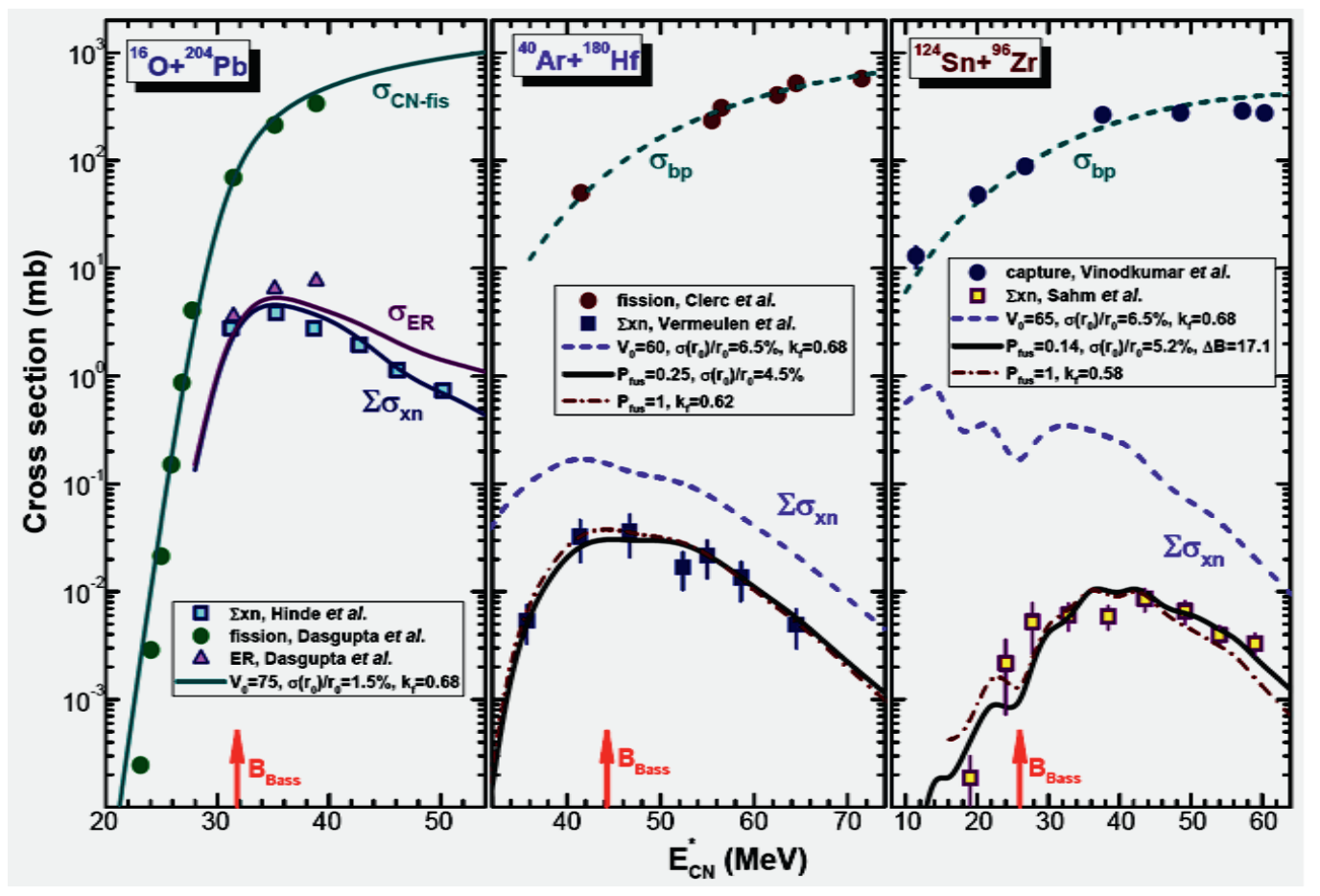

Fig. 4. Fission (capture) and ER cross sections obtained in the ${ }^{16} \mathrm{O}+{ }^{204} \mathrm{~Pb}[34],{ }^{40} \mathrm{Ar}+{ }^{180} \mathrm{Hf}[36,36]$ and ${ }^{124} \mathrm{Sn}+{ }^{96} \mathrm{Zr}[9,37]$ reactions leading to the same ${ }^{220} \mathrm{Th}^{*} \mathrm{CN}$ (symbols). Calculations without any fusion suppression and with fission barriers obtained from the ${ }^{16} \mathrm{O}+{ }^{204} \mathrm{~Pb}$ data fit are shown by dashed lines for the ${ }^{40} \mathrm{Ar}$ and ${ }^{124} \mathrm{Sn}$ induced reactions, whereas similar calculations with the adjusted $P_{\text {fus }}$ values are shown by solid lines for ER cross sections. Alternative fits corresponding to $P_{f u s}=1$ and smaller fission barriers $\left(k_{f}\right.$ values) are shown by dash-dotted lines. See the text for further details.

More neutron-deficient Th compound nuclei can be only produced in reactions with massive nuclei. Their production in a very asymmetric combination, which is similar to the ${ }^{16} \mathrm{O}+{ }^{204} \mathrm{~Pb}$ one, is impossible. Therefore, a simple extrapolation with the proportionality of the $k_{f}$ values, corresponding to the data fits with and without fusion suppression for similar reactions leading to the ${ }^{220} \mathrm{Th}^{*} \mathrm{CN}$ could be used. This extrapolation gives us considerably higher magnitudes of macroscopic fission barriers than one can get with a straight fit of calculations to the measured ER cross sections. For example, in the case of reactions with the ${ }^{32} \mathrm{~S}[38,39],{ }^{40} \mathrm{Ar}[36]$ and ${ }^{60} \mathrm{Ni}$ [39] projectiles leading to the ${ }^{214,216} \mathrm{Th}^{*}$ compound nuclei, straightforward fits give us $k_{f}=0.56$ comparing to the $k_{f}=0.614$ value obtained with the extrapolation. Using this scaling one can obtain fusion probability values (with some dependence on the extrapolated $k_{f}$ values) and reproduce ER cross sections measured in the investigated reactions leading to the neutron-deficient Th compound nuclei.

Some examples of fusion probabilities obtained with this empirical approach for reactions leading to the production of Th compound nuclei are shown in Figure 5. In the case of the ${ }^{214} \mathrm{Th}^{*} \mathrm{CN}$ one can compare the present data with the fusion probabilities extracted from the fission data [38] and 


\section{$\mathrm{CNR} * 11$}

calculated with the DNS model [40]. It should be stressed that the resulting fusion probability values at energies well above the fusion barrier are noticeably smaller than unity in contrast to the previous studies for similar reactions $[9,41]$. This is the result of the normalization of experimental ER cross sections to the calculation with fission barriers obtained in the analysis of very asymmetric reactions. To shed light on the effect of static deformations of fusioning nuclei, one should consider relative positions of the fusion barriers corresponding to 'tip' and 'side' collisions for projectile and target nuclei (see Figure 5). These positions (relatively to the Bass barrier [28]) were calculated according to [21] using the nuclear proximity potential and tabulated deformation parameters [17] for projectile and target nuclei. As one can see from the figure, no evident correlations are observed between the barrier spans corresponding to their minimal and maximal magnitudes and slopes of fusion probability curves in the vicinity of the nominal fusion barrier [28]. We see an expected growth of the fusion probability in going from 'tip' to 'side' configurations due to the reduction in the corresponding distance between fusioning nuclei.

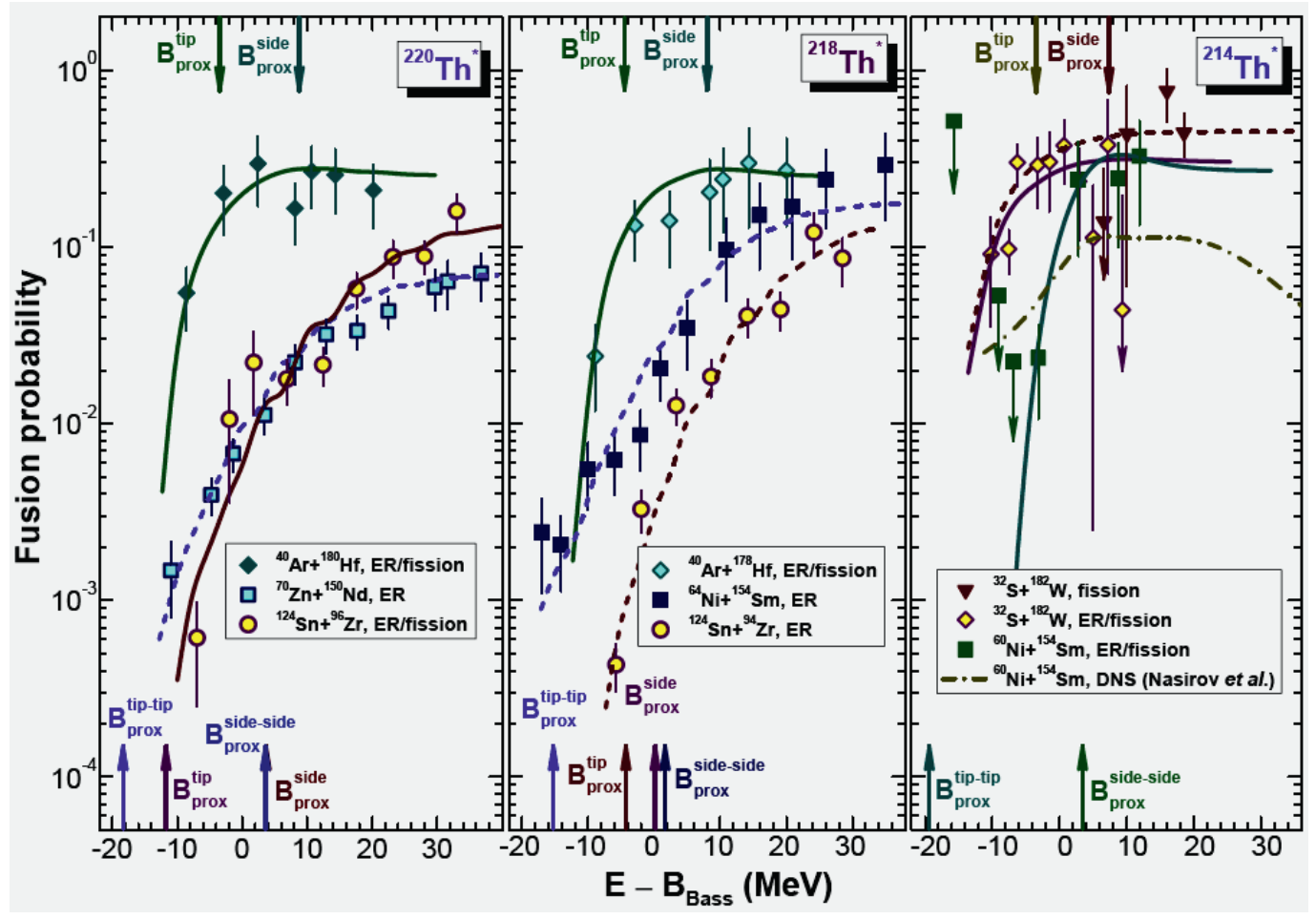

Fig. 5. Fusion probabilities obtained as the ratio of the experimental ER cross sections $\Sigma \sigma_{x n}$ obtained in [36] $\left({ }^{40} \mathrm{Ar}\right)$, [39] $\left.{ }^{32} \mathrm{~S},{ }^{60,64} \mathrm{Ni}\right),[42]\left({ }^{70} \mathrm{Zn}\right)$ and [9] $\left({ }^{124} \mathrm{Sn}\right)$ to the calculated ones for reactions leading to the ${ }^{220,218,214} \mathrm{Th}^{*}$ compound nuclei (symbols with error bars). Solid lines correspond to the calculations based on the fit of barrier-passing cross sections to the capture ones measured in [36] $\left({ }^{40} \mathrm{Ar}+{ }^{180,178} \mathrm{Hf}\right),[37]\left({ }^{124} \mathrm{Sn}+{ }^{96} \mathrm{Zr}\right)$ and $[38,39]\left({ }^{32} \mathrm{~S}+{ }^{182} \mathrm{~W}\right)$, whereas dashed lines reproduce the calculations based on the assumed capture cross sections. For the latter the same parameter values are used as obtained for similar reactions at the fit of the calculated barrier-passing cross section.

One should mention that the similar analysis of reactions leading to Po compound nuclei shows the fusion probabilities approaching the unity at energies well above the fusion barrier [43]. In this study we also extracted the macroscopic component of fission barriers in a wide region of neutron numbers, which were compared with predictions of different models and tabulated values. In the same way the macroscopic components of fission barriers for the heavier nuclei from Rn to Th were obtained, but in this case only very asymmetric combinations were considered in the SM analysis. 
These data are shown in Figure 6 together with the predictions of different models [30, 44-47] and tabulated values [48] for the macroscopic barriers (bottom panels). Shell corrections calculated in [17] and those obtained in the framework of the present analysis (see Section 2) could be also compared (upper panels). As one can see, the extracted macroscopic fission barriers for neutron-deficient nuclei are lower than any model calculations, with the exception of the predictions given by the LSD model [47]. At the same time, the values for relatively neutron-rich Ra and Th nuclei approach to the tabulated ones [48], as observed for Po nuclei [43].

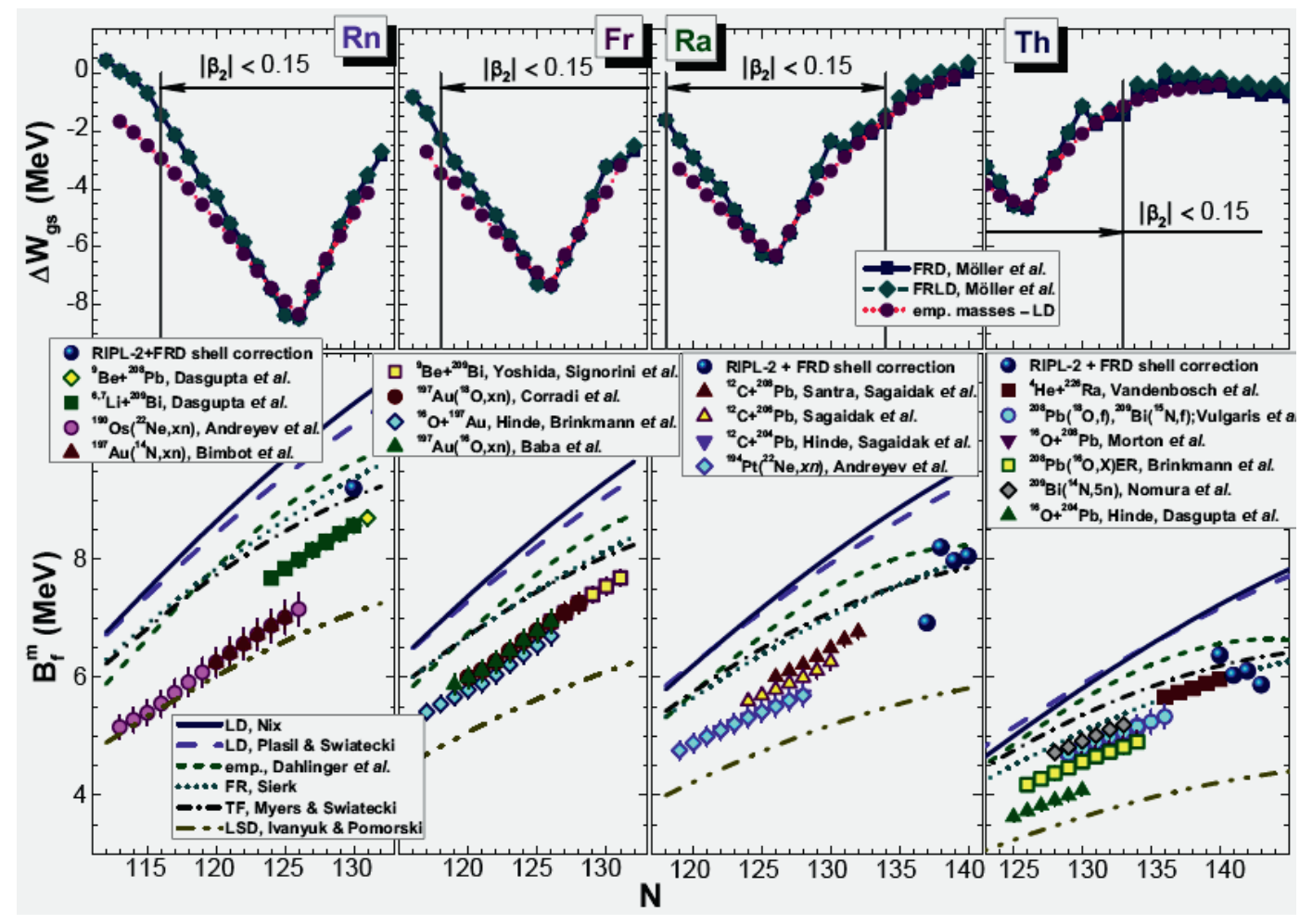

Fig. 6. Macroscopic fission barriers derived with the SM analysis of the ER and fission cross sections measured in very asymmetric reactions leading to the $\mathrm{Rn}^{*}, \mathrm{Fr}^{*}, \mathrm{Ra}^{*}$ and $\mathrm{Th}^{*}$ compound nuclei (different symbols with error bars correspond to the observed $\mathrm{CN}$ evaporation chains). Lines correspond to the LD [30], empirical [44], finite-range [45] (FR), Thomas-Fermi [46] (TF) and Lublin-Strasbourg drop [47] (LSD) model calculations performed for these nuclei (bottom panels). Refs to the experimental data obtained in reactions leading to the $\mathrm{Rn}^{*}, \mathrm{Fr}^{*}, \mathrm{Ra}^{*}$ and $\mathrm{Th}^{*}$ compound nuclei can be found in [49], [50], [12, 33] and [34, 49], respectively. Shell corrections obtained in the present SM analysis are compared with the ones tabulated for the finite range droplet (FRD) and finite range liquid drop (FRLD) models [17]. The region of near-spherical nuclei corresponding to the deformation parameter $\left|\beta_{2}\right| \leq 0.15$ [17] is indicated (upper panels).

\section{Heaviest nuclei production}

In contrast to the description of Po to Th nuclei production, the same SM analysis shows that Fm excitation functions measured in very asymmetric reactions can be reproduced with the scaled LD fission barriers which are about 20\% higher than the original ones [51]. Applying these LD barriers to the production of Fm isotopes in the 'cold' fusion reaction, one can obtain the averaged fusion probability value about $14 \%$ allowing to reproduce the $x n$ evaporation cross sections measured in the ${ }^{40} \mathrm{Ar}+{ }^{208} \mathrm{~Pb}$ reaction [52]. This value is comparable with $P_{f u s}=(32 \pm 17) \%$ derived from the fission experiment carried out at a higher energy [53]. In the case of the Fm production in the more symmetric 


\section{$\mathrm{CNR} * 11$}

${ }^{50} \mathrm{Ti}+{ }^{198} \mathrm{Pt}$ combination with the weakly deformed target nucleus we come to more severe fusion suppression than in the previous case. The ${ }^{50} \mathrm{Ti}+{ }^{198} \mathrm{Pt}$ data [52] can be described with the $1.5 \%$ value of the fusion probability together with the additional $\Delta B=11.3 \mathrm{MeV}$ energy to the fusion barrier, which is introduced according to the EEP formalism, similar to the way it was done in the case of ${ }^{124} \mathrm{Sn}+{ }^{96} \mathrm{Zr}$ (see above). Resulting values of the fusion probability for both reactions are shown in Figure 7 that demonstrates essentially different dependences of $P_{f u s}$ on energy for 'cold' fusion reactions induced on spherical and deformed target nuclei.

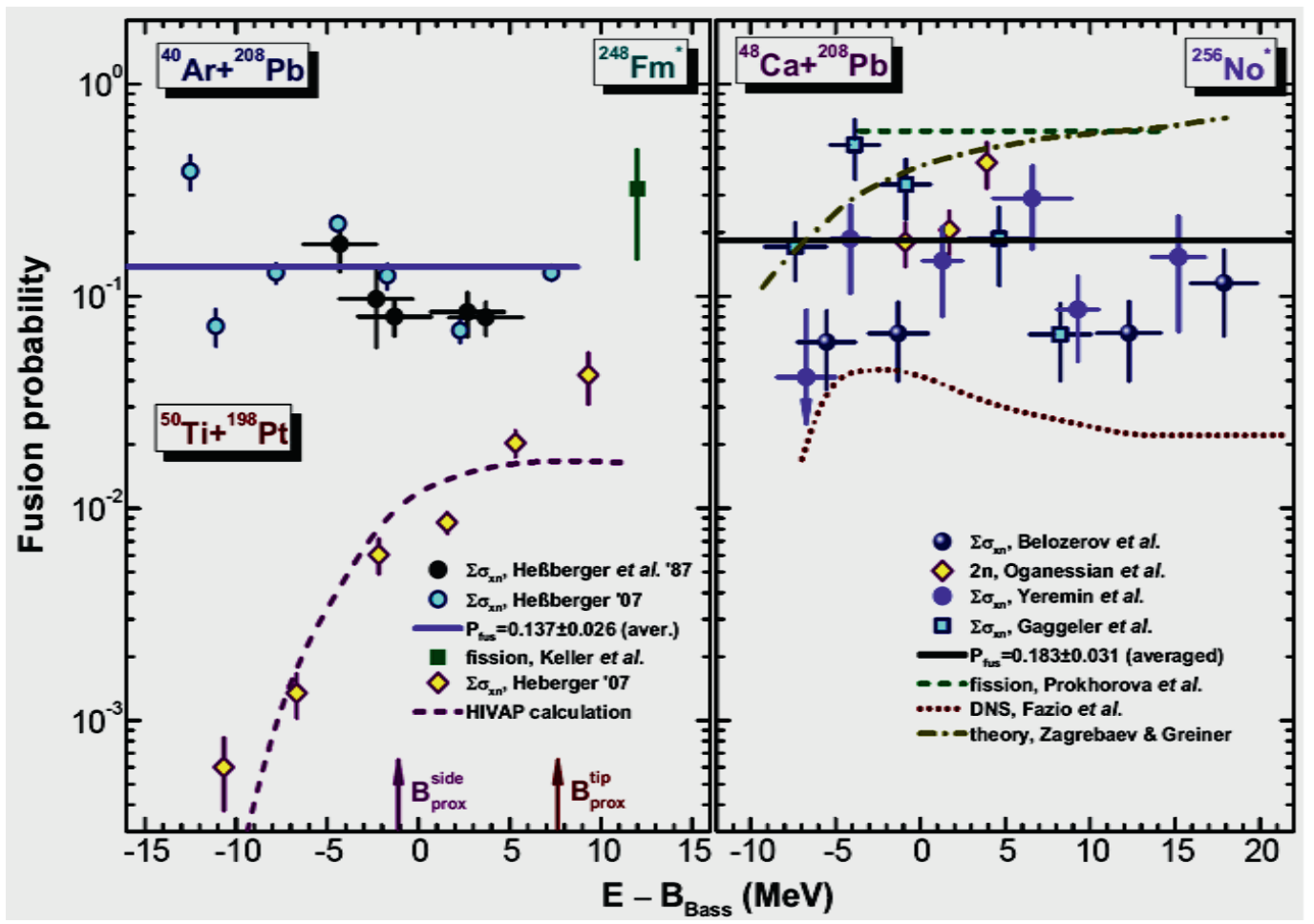

Fig. 7. Fusion probabilities derived as the ratio of the experimental ER cross sections $\sum \sigma_{x n}$ obtained in the ${ }^{40} \mathrm{Ar}+{ }^{208} \mathrm{~Pb},{ }^{50} \mathrm{Ti}+{ }^{198} \mathrm{Pt}$ and ${ }^{48} \mathrm{Ca}+{ }^{208} \mathrm{~Pb}$ reactions [52, 55 and Refs therein] to the calculated ones (symbols with error bars). Solid line corresponds to the averaged $P_{\text {fus }}$ value for ${ }^{40} \mathrm{Ar}+{ }^{208} \mathrm{~Pb}$ and ${ }^{48} \mathrm{Ca}+{ }^{208} \mathrm{~Pb}$, whereas a dashed line in the left panel corresponds to the calculation fitted to the measured ${ }^{50} \mathrm{Ti}+{ }^{198} \mathrm{Pt}$ ER cross sections. $P_{\text {fus }}$ values extracted from fission experiments $[53,56]$ and obtained with theoretical models $[57,58]$ are also shown. See the text for details.

The SM analysis of the No isotopes production in the ${ }^{12,13} \mathrm{C}+{ }^{244,246,248} \mathrm{Cm}$ reactions [54] also shows that the LD fission barriers should be $20 \%$ higher than the nominal ones in order to describe excitation functions for the production of these nuclei. Using these macroscopic fission barriers for the analysis of the ER and fission cross sections obtained in the ${ }^{48} \mathrm{Ca}+{ }^{208} \mathrm{~Pb}$ reaction (see [55] and [56], respectively, and Refs therein), one can come to the similar result as obtained for ${ }^{40} \mathrm{Ar}+{ }^{208} \mathrm{~Pb}$. Namely, the averaged value of $P_{f u s} \approx 18 \%$ can be derived with the macroscopic fission barriers obtained in the SM analysis of the $x n$ excitation functions measured for the $\mathrm{C}+\mathrm{Cm}$ reactions. This value is significantly lower than the $P_{\text {fus }}$ values extracted from the fission data [56] and obtained with the theory [57] but is much higher than it follows from the DNS model calculation [58], as shown in Figure 7. The $P_{f u s}$ values obtained in the present analysis of 'cold' fusion reactions slightly differ from those estimated earlier in the preliminary study [51]. One should also mention that the alternative independent fits to both sets of the data give us significantly smaller LD fission barriers corresponding to $k_{f}=0.9$ and 0.8 for ${ }^{40} \mathrm{Ar}+{ }^{208} \mathrm{~Pb}$ and ${ }^{48} \mathrm{Ca}+{ }^{208} \mathrm{~Pb}$, respectively, [51], i.e., the values that contradict the result of the very asymmetric reactions analysis, which yields $k_{f}=1.2$. 
The same LD fission barriers scaling can be obtained for the relatively neutron-rich Rf isotopes produced in very asymmetric combinations with ${ }^{15} \mathrm{~N}$ and ${ }^{16,18} \mathrm{O}$ (see [51] and Refs therein). Applying this result to the data obtained in less asymmetric combinations with ${ }^{22} \mathrm{Ne}$ and ${ }^{26} \mathrm{Mg}$ leading to the same ${ }^{264} \mathrm{Rf}^{*} \mathrm{CN}$, we get some overestimates for the $x n$ evaporation cross sections in calculations. At the same time, the ${ }^{26} \mathrm{Mg}+{ }^{238} \mathrm{U}$ ER cross sections [59] can be better described using the fusion probability extracted from the fission data [7]. The analysis of ratios of the $\mathrm{CN}$-fission to capture cross sections obtained in the ${ }^{238} \mathrm{U}$ induced reaction on heavier targets [7] shows the decrease in the fusion probability with $Z$ of a target nucleus without any dependence on the energy. The last statement is not fulfilled in the case of the ${ }^{48} \mathrm{Ca}+{ }^{238} \mathrm{U}$ fission data $[7,60]$ for which decrease in the fusion probability is observed even at energies above the nominal fusion barrier [28]. In further study one can use the systematics of the $P_{\text {fus }}$ values derived from the fission data $[6,7]$ corresponding to energies above the nominal fusion barrier [28]. The $P_{f u s}$ values demonstrate an exponential dependence on the Coulomb factor as shown in Figure 8. The energy dependence of $P_{f u s}$ at barrier and sub-barrier energies reveal the data obtained with ER cross sections measured in the ${ }^{26} \mathrm{Mg}$,

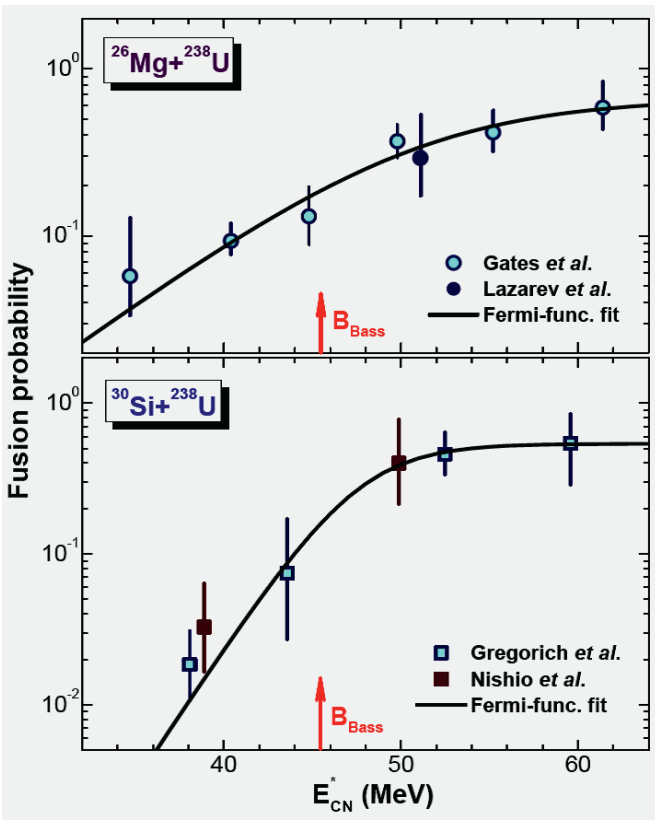

Fig. 9. Fusion probabilities for the ${ }^{26} \mathrm{Mg},{ }^{30} \mathrm{Si}+{ }^{238} \mathrm{U}$ reactions, which are derived as the ratio of the measured $\Sigma \sigma_{x n}$ cross sections $[59,61]$ to those calculated as described in the text (symbol with error bars). Fermi-function fits to the data are shown by solid lines. See details in the text.

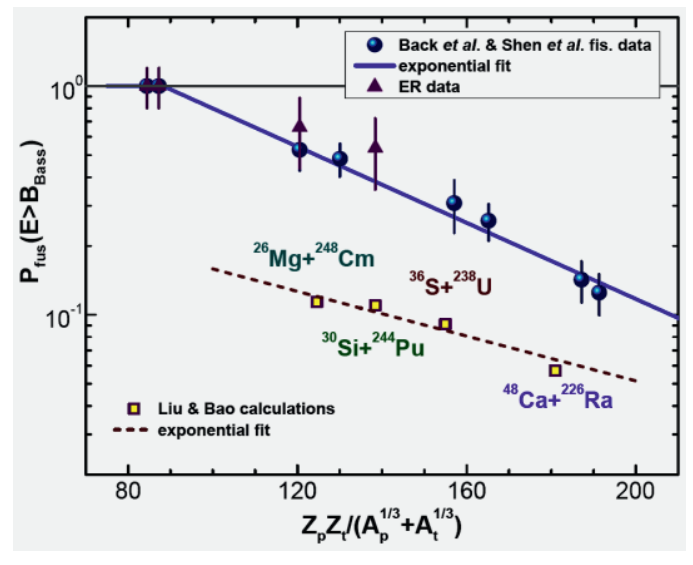

Fig. 8. Fusion probabilities derived from the ratios of the $\mathrm{CN}$-fission cross sections to those for capture, which obtained in $[6,7]$ for deformed nuclei at energies above the Bass barrier [28] (spheres with error bars). Asymptotic values of $P_{\text {fus }}$ derived from Fermi-function fits to the ${ }^{26} \mathrm{Mg},{ }^{30} \mathrm{Si}+{ }^{238} \mathrm{U}$ data and values corresponding to very asymmetric reactions leading to the ${ }^{264} \mathrm{Rf}^{*} \mathrm{CN}$ (see Refs in [51]) are shown by filled triangles with error bars. The $P_{\text {fus }}$ values obtained with the diffusion model [62] for reactions leading to the ${ }^{274} \mathrm{Hs}^{*} \mathrm{CN}$ are also shown by squares.

${ }^{30} \mathrm{Si}+{ }^{238} \mathrm{U}$ reactions $[59,61]$. These data are shown in Figure 9. They are derived from the ratios of the $\Sigma \sigma_{x n}$ measured in the evaporation reactions to the calculated ones obtained with the capture cross sections measured in the same reactions. Asymptotic values of $P_{\text {fus }}$ corresponding to the fusion probability at energies well above the fusion barrier are estimated with the Fermi-function fits to the data. As one can see in Figure 8 these values do not contradict to the ones derived from fission data. Similar fits were used to estimate the asymptotic values of $P_{\text {fus }}$ for the ${ }^{48} \mathrm{Ca}+{ }^{238} \mathrm{U}$ and ${ }^{36} \mathrm{~S}+{ }^{238} \mathrm{U}$ data obtained in the fission experiments $[7,60,63]$. The value extracted from the data [7] is included into the systematics shown in Figure 8. Similar values extracted from the data $[60,63]$ obtained recently are not included into the systematics, but they are in agreement with it.

The application of this systematics to the analysis of the ER data obtained in the ${ }^{34} \mathrm{~S}+{ }^{238} \mathrm{U}$ reaction [64] leading to the ${ }^{272} \mathrm{Hs}^{*} \mathrm{CN}$ shows a good agreement of calculations with the meas- 


\section{$\mathrm{CNR} * 11$}

ured ER cross sections, which is achieved using the LD fission barriers without any scaling. At the same time, the description of the data with zero LD fission barriers and fusion probability equalled to unity seems to be also acceptable. The analysis of the $\mathrm{Hs}$ isotopes production in the ${ }^{26} \mathrm{Mg}+{ }^{248} \mathrm{Cm}$ and ${ }^{48} \mathrm{Ca}+{ }^{226} \mathrm{Ra}$ reactions $[65,66]$ leading to the same ${ }^{274} \mathrm{Hs}^{*} \mathrm{CN}$ may clarify this situation. Thus, options with the macroscopic barriers equalled to the LD ones and to zero ones do not fit both the data, whereas the option with the half of LD barriers and with the fusion probabilities corresponding to the systematics shown in Figure 8 satisfies both the data as shown in Figure 10. The only exception is the $3 n$ cross section data obtained in the ${ }^{26} \mathrm{Mg}+{ }^{248} \mathrm{Cm}$ reaction at deep sub-barrier energies [65]. To describe them one has to use a very large value of the fusion barrier fluctuation parameter $\sigma\left(r_{0}\right) / r_{0}$ (see Section 2) which is not needed in the description of the heaviest nuclei production in the ${ }^{26} \mathrm{Mg}$ and ${ }^{30} \mathrm{Si}$ induced reactions on ${ }^{238} \mathrm{U}[59,61]$. One should mention in this connection that the isotope ${ }^{271} \mathrm{Hs}$ being the product of the $3 n$ evaporation channel was not observed in our ${ }^{48} \mathrm{Ca}+{ }^{226} \mathrm{Ra}$ experiment [66]. Thus, the unexpected enhancement of the $3 n$ evaporation channel in the ${ }^{26} \mathrm{Mg}+{ }^{248} \mathrm{Cm}$ reaction needs further confirmations.

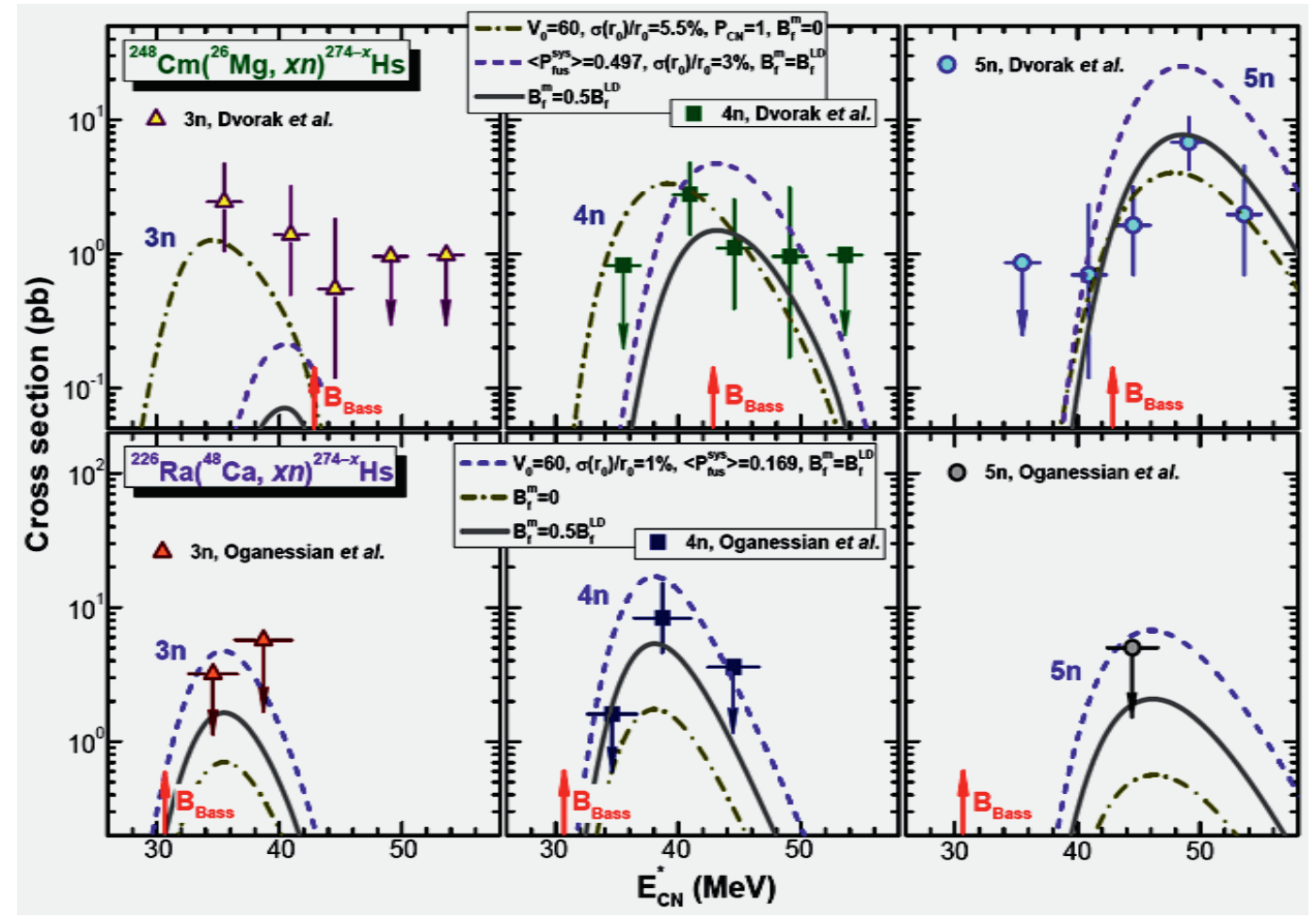

Fig. 10. ER cross sections obtained in the ${ }^{26} \mathrm{Mg}+{ }^{248} \mathrm{Cm}$ [65] and ${ }^{48} \mathrm{Ca}+{ }^{226} \mathrm{Ra}$ [66] reactions leading to the same ${ }^{274} \mathrm{Hs}^{*} \mathrm{CN}$ (symbols). Calculations with fusion suppression (the $P_{\text {fus }}$ values are taken from the systematics presented in Figure 8) and without it along with a different scaling for the LD fission barriers are shown by different lines for both the reactions. See the text for details.

Let us consider a choice of fission barriers in calculations for the heaviest nuclei. As shown in numerous studies as well as in the present analysis, fission barriers are the most indefinite input parameter determining the survivability of the heaviest nuclei in calculations. The simple scaling expressed by Eq. (6) was usually used in a number of previous studies [10, 12, 26, 33, 43, 49-51, 55]. The necessity of such scaling stems from the fact that model calculations demonstrate very different values of the macroscopic component of fission barriers as shown in Figure 6. The same is at the end of nuclear stability for the heaviest nuclei. At the same time, this component disappears in going from $\mathrm{Hs}$ to $\mathrm{Cn}$ nuclei according to the model calculations [30, 44-47]. So fission barriers for the 
elements heavier than $\mathrm{Cn}$ are determined by the shell corrections only or by the difference between the empirical [31] and LD (macroscopic) masses [32]. The comparison of the calculated Finite Range Droplet (FRD), Finite Range Liquid Drop (FRLD) [17], Thomas-Fermi (TF) [67] and Hartree-Fock-Bogoliubov (HFB-2) [68] masses, with the empirical ones (AWT) [31] for isotopes of the elements with $Z=110$ to 118 shows that the FRLD masses are the best ones from the point of view of their agreement with the empirical ones, whereas the FRD masses announced as the best ones in [17] are systematically shifted to the lower $(M-A)$-values and seem to be the worst ones. The values of TF and HFB-2 masses are somewhere between the predictions of the FRD and FRLD models. A quantitative analysis confirms this statement. The sums over the number of isotopes $n$ tabulated in [31] for root mean square deviations of the calculated masses from the empirical ones have the smallest values for the FRLD masses [17] for the elements with $Z=110-118$, as one can see in Figure 11. Thus, one can recommend using the FRLD masses for further calculations.

In Figure 12 the measured cross sections for isotopes of the elements of $\mathrm{Cn}$ and 114 produced in fusion-evaporation reactions induced by ${ }^{48} \mathrm{Ca}$ [69] are shown and compared with the excitation functions calculated using the FRLD masses and the systematics of fusion probability presented in Figure 8. The EEP formalism (see above) was also applied to reproduce the measured cross sections. Similar comparison was also made for the production of isotopes of the elements of 116 and 118 in the reactions induced by ${ }^{48} \mathrm{Ca}$ [70]. These comparisons show that production of the heaviest nuclei close to the region of superheavy elements could be quite satisfactory reproduced in the framework of the SM calculations using the FRLD masses for the estimates of fission barrier heights $\left(B_{f}=\Delta W_{g s}\right)$ for nuclei involved in an evaporation-fission cascade. These input quantities determine mainly the calculated survivability for the heaviest nuclei produced in fusionevaporation reactions.

Another point is the fusion suppression that imposes a constraint on the $\mathrm{CN}$ formation in the entrance reaction channel. This constraint could be reproduced in calculations by the corre-

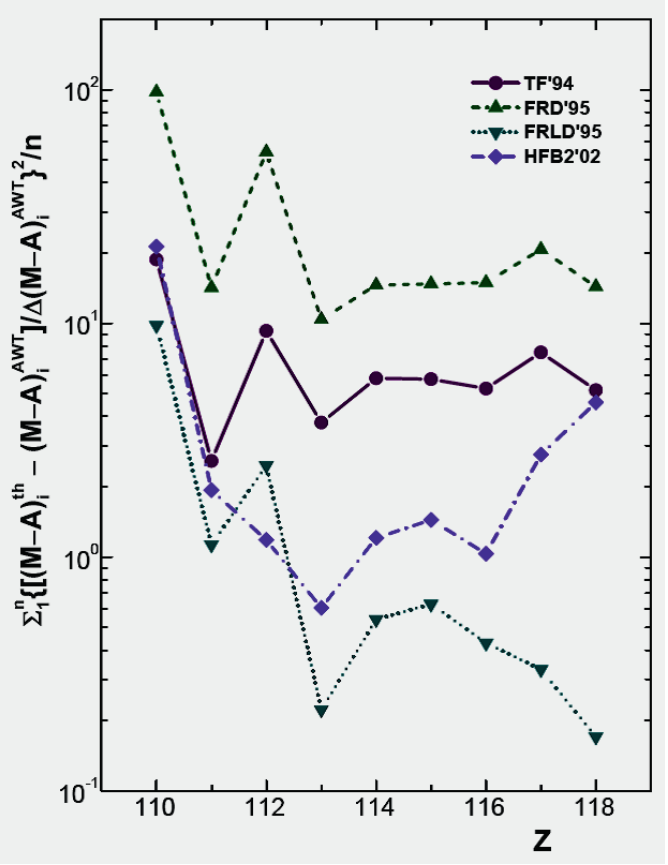

Fig. 11. The sum of root mean square deviations of the calculated masses from empirical ones [31] for the FRD, FRLD [17], TF [67] and HFB-2 [68] masses for isotopes of elements of $Z=110-118$. See designations in the text. sponding function of the fusion probability. The asymptotic values of this functions (fusion probabilities at energies well above the fusion barrier) could be determined with the empirical systematics shown in Figure 8. Besides this limitation the width of the fusion barrier distribution $\sigma\left(r_{0}\right) / r_{0}$ (see Section 2) should be significantly decreased to reproduce the data shown in Figure 12. In fact, $\sigma\left(r_{0}\right) / r_{0}$ is the only parameter varied within $(0-1.5) \%$ to describe the sub-barrier $2 n$ and $3 n$ evaporation cross sections. These values are much lower than the ones that are inherent in reactions with massive and deformed reactants leading to the nuclei near the $N=126$ shell and show $\sigma\left(r_{0}\right) / r_{0}=(4-5) \%$, as indicated in Figure 4 and in Refs. $[9,10,12,33,36$, 43, 49-51]. Fusion suppression at barrier and sub-barrier energies could be also reproduced in the framework of the extra-extra-push formalism using parameter values obtained in [7]. In Figure 12 this variant of calculations demonstrates a similar agreement with the experimental data. This set of parameter values give a corresponding addition to the fusion barrier of reactions $\Delta B$ indicated in the figure. Such increase in the height of the fusion barrier is accompanied by some increase in the width 
of the fusion barrier distribution corresponding to $\sigma\left(r_{0}\right) / r_{0}=(1.5-3) \%$, which should be used to describe the data (see Figure 12).

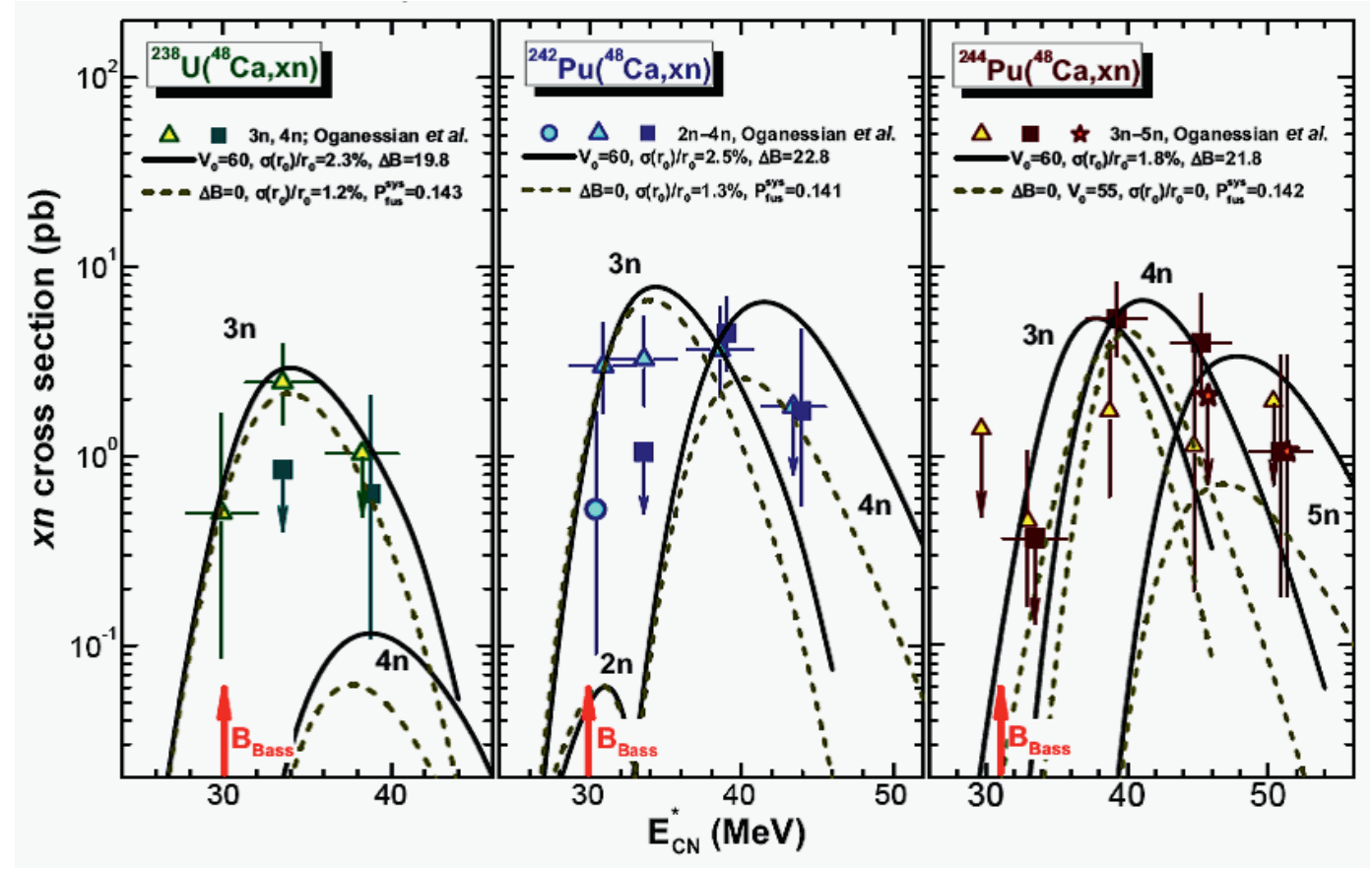

Fig. 12. The $x n$ evaporation cross sections obtained in the ${ }^{48} \mathrm{Ca}+{ }^{238} \mathrm{U}$ and ${ }^{48} \mathrm{Ca}+{ }^{242,244} \mathrm{Pu}[69]$ reactions leading to the ${ }^{286} \mathrm{Cn}^{*}$ and ${ }^{290,292} 114$ compound nuclei (symbols). Calculations with the $P_{\text {fus }}$ values taken from the systematics presented in Figure 8 and with the EEP formalism [7] are shown by dashed and solid lines, respectively. In both variants of calculations the FRLD masses [17] were used for the estimates of fission barrier heights. See the text for details.

\section{Summary and conclusion}

Being critical and correlating values in the estimates of evaporation residue cross sections, fusion probability and survivability in fusion-evaporation reactions leading to heavy and heaviest nuclei were considered using the potential barrier-passing model for capture of nuclei and statistical model (SM) for the compound nucleus de-excitation.

The survivability of heavy nuclei produced in the vicinity of a 126 neutron shell in very asymmetric projectile-target combinations can be reproduced with reduced values of the liquid-drop (LD) component of fission barriers in the framework of SM. These barriers can be used for the empirical estimates of the fusion probability for more symmetric reactions.

Production of heavy nuclei from Fm to Rf in very asymmetric 'hot' fusion reactions can be reproduced with the $20 \%$ increase in the LD component of fission barriers with the use of a similar SM approach. The corresponding estimates of the fusion probability for more symmetric 'cold' fusion reactions differ from those predicted by theories and obtained in fission experiments.

Production of the heaviest nuclei with $Z>104$ in 'hot' fusion reactions induced by massive projectiles can be reproduced with the fusion probabilities obtained from fission data and with some reduction in the LD component of fission barriers (for Hs nuclei). Data analysis implies a disappearance of the macroscopic (LD) component for nuclei with $Z>108$. 
Estimates of atomic masses and shell corrections are mainly responsible for the description of the heaviest nuclei production in reactions induced by ${ }^{48} \mathrm{Ca}$ and heavier projectiles, bearing in mind an absence of the macroscopic component in the fission barriers for these nuclei.

\section{Acknowledgments}

This work was supported by the Russian Foundation for Basic Research (Grants No.11-02-12050 and No.11-02-12066).

\section{References}

1. S. Hofmann, G. Münzenberg, Rev. Mod. Phys. 72, 733 (2000)

2. Yu. Oganessian, J. Phys. G 34, R165 (2007)

3. W. J. Swiatecki, Phys. Scr. 24, 113 (1981)

4. R. Bock et al., Nucl. Phys. A 338, 334 (1982)

5. J. Tōke et al., Nucl. Phys. A 440, 327 (1985)

6. B.B. Back, Phys. Rev. C 31, 2104 (1985); B.B. Back et al., Phys. Rev. C 32, 195 (1985)

7. W.Q. Shen et al., Phys. Rev. C 36, 115 (1987)

8. W.U. Schröder, J.R. Huizenga, Damped Nuclear Reactions, Treatise on Heavy-Ion Science (Plenum Press, New York, 1984), Vol. 2, p. 115

9. C.-C. Sahm et al., Nucl. Phys. A 441, 316 (1985)

10. A.B. Quint et al., Z. Phys. A 346, 119 (1993)

11. A.C. Berriman et al., Nature 413, 144 (2001); D.J. Hinde et al., J. Nucl. Radiochem. Sci. 3, 31 (2002)

12. R.N. Sagaidak et al., Phys. Rev. C 68, (2003) 014603

13. K.T.R. Davies, A.J. Sierk, Phys. Rev. C 31, 915 (1985)

14. U.L. Businaro, S. Gallone, Nuovo Cimento 1, 629 (1955); ibid. 1277 (1955)

15. G. Royer, B. Remaud, Nucl. Phys. A 444, 477 (1985)

16. R. Moustabchir, G. Royer, Nucl. Phys. A 683, 266 (2001)

17. P. Mőller, et al., At. Data Nucl. Data Tables 59, 185 (1995)

18. G.G. Adamian et al., Phys. Rev. C 69, 011601R (2004); ibid. 046401 (2004)

19. G. Fazio et al., Eur. Phys. J. A 19, 89 (2004)

20. V.I. Zagrebaev, Phys. Rev. C 64, 034606 (2001)

21. V.I. Zagrebaev et al., http://nrv.jinr.ru/nrv/webnrv/

22. R.S. Naik et al., Phys. Rev. C 76, 054604 (2007)

23. G.N. Knyazheva et al., Phys. Rev. C 75, 064602 (2007)

24. A.M. Stefanini et al., Eur. Phys. J. A 23, 473 (2005)

25. G. Mandaglio et al., The $2^{\text {nd }}$ International Conference on Current Problems in Nuclear Physics and Atomic Energy, Kiev, Ukraine, June 9-15, 2008. Part I, p. 187

26. W. Reisdorf et al., Nucl. Phys. A 438, 212 (1985)

27. C.H. Dasso, S. Landowne, and A. Winther, Nucl. Phys. A 407, 221 (1983)

28. R. Bass, Phys. Rev. Lett. 39, 265 (1977); Lect. Notes Phys. 117, 281 (1980)

29. W. Reisdorf, Z. Phys. A 300, 227 (1981); W. Reisdorf, M. Schädel, Z. Phys. A 343, 47 (1992)

30. S. Cohen, F. Plasil, W.J. Swiatecki, Ann. Phys. (NY) 82, 557 (1974)

31. G. Audi, A.H.Wapstra, C. Thibault, Nucl. Phys. A 729, 337 (2003)

32. W. D. Myers, W. J. Swiatecki, Ark. Fys. 36, 343 (1967)

33. R.N. Sagaidak et al., The 10th International Conference on Nuclear Reaction Mechanisms, Varenna, Italy, June 12-16, 2003, edited by E. Gadioli (Universitá degli Studi di Milano, Milan, 2003), p. 301; JINR Preprint E7-2003-149, Dubna, 2003

34. M. Dasgupta et al., Rhys. Rev. Lett. 99, 192701 (2007)

35. H.-G Clerc et al., Nucl. Phys. A 419, 571 (1984)

36. D. Vermeulen et al., Z. Phys. A 318, 157 (1984) 


\section{$\mathrm{CNR} * 11$}

37. A.M. Vinodkumar et al., Phys. Rev. C 74, 064612 (2006)

38. J.G. Keller et al., C 36, 1364 (1987)

39. S. Mitsuoka et al., Phys. Rev. C 62, 054603 (2000); ibid., Phys. Rev. C 65, 054608 (2002)

40. A. Nasirov et al., Nucl. Phys. A 759, 342 (2005)

41. K. Nishio et al., Phys. Rev. C 63, 044610 (2001); K. Satou et al., Phys. Rev. C 65, 054602 (2002)

42. C. Stodel, THESE Docteur de l'Université de Caen, LPCC T 98-05 (1998)

43. R.N. Sagaidak, A.N. Andreyev, Phys. Rev. C 79, 054613 (2009)

44. M. Dahlinger et al., Nucl. Phys. A 376, 94 (1982)

45. A.J. Sierk, Phys. Rev. C 33, 2039 (1986)

46. W.D. Myers, W.J. Świątecki, Phys. Rev. C 60014606 (1999)

47. F.A. Ivanyuk, K. Pomorski, Phys. Rev. C 79054327 (2009)

48. T. Belgya et al., RIPL-2 (IAEA, Vienna, 2006), http://www-nds.iaea.org/RIPL-2/

49. R.N. Sagaidak, Nuclear Structure and Dynamics '09, Dubrovnik, Croatia, May 4-8, 2009, edited by M. Milin et al., AIP Conf. Proc. 1165, 379 (2009)

50. L. Corradi et al., Phys. Rev. C 71014609 (2005)

51. R.N. Sagaidak, Eur. Phys. J. D 45, 59 (2007)

52. F.P. Heßberger et al., GSI Scientific Report 1986, GSI 87-1, p. 17; F.P. Heßberger, Eur. Phys. J. D 45, 33 (2007)

53. H. Keller et al., Z. Phys. A 326, 313 (1987)

54. T. Sikkeland et al., Phys. Rev. 172, 1232 (1968)

55. A.V. Belozerov et al., Eur. Phys. J. A 16, 447 (2003)

56. E.V. Prokhorova et al., Nucl. Phys. A 802, 45 (2008)

57. V. Zagrebaev, W. Grainer, Phys. Rev. C 78, 034610 (2008)

58. G. Fazio et al., Mod. Phys. Lett. A 20, 391 (2005)

59. Yu.A. Lazarev et al., Phys. Rev. C 62, 064307 (2000); J.M. Gates et al., Phys. Rev. C 77, 034603 (2008)

60. E.M. Kozulin et al., Phys. Lett. B 686, 227 (2010)

61. K.E. Gregorich et al., Phys. Rev. C 74, 044611 (2006); K. Nishio et al., Eur. Phys. J. A 29, 281 (2006)

62. Z.H. Liu, Jing-Dong Bao, Phys. Rev. C 74, 057602 (2006)

63. I.M. Itkis et al., Phys. Rev. C 83, 064613 (2011)

64. Yu.A. Lazarev et al., Phys. Rev. Lett. 75, 1903 (1995); K. Nishio et al., Phys. Rev. C 81, 061601(R) (2010)

65. J. Dvorak et al., Phys. Rev. Lett. 100, 132503 (2008)

66. Yu.Ts. Oganessian et al., to be published

67. W.D. Myers, W. J. Swiatecki, Nucl. Phys. A 601141 (1996); http://ie.lbl.gov/txt/ms.txt

68. S. Goriely et al., Phys. Rev. C 66, 024326 (2002); http://www-astro.ulb.ac.be

69. Yu.Ts. Oganessian et al., Phys. Rev. C 70, 064609 (2004)

70. Yu.Ts. Oganessian et al., Phys. Rev. C 74, 044602 (2006) 